\title{
Influence of low stress handling during clinical visit on physiological and behavioural indicators in adult dogs: a preliminary study
}

\author{
Bruno Scalia, Daniela Alberghina and Michele Panzera
}

Low stress handling techniques or "Fear Free principles" in veterinary clinics are becoming an important research area aimed at improving small animal welfare, considering that the majority of dogs who undergo clinical examinations exhibit fear or anxiety signs. Objective of this study was to compare a number of physiological and behavioural indicators using low stress handling (LSH) and traditional (TT) techniques in order to assess whether the LSH approach had a positive impact on the dog's welfare. Eight adult dogs were filmed while undergoing both LSH and TT visits (separated by a distance of seven weeks). The same usual sequence of events was followed for both visits (e. g. muzzle wearing, heart and lungs stethoscope examination, etc.) except that 1) during the LSH visit, the dog was free to explore the environment (while receiving treats) and play for five minutes before and after the visit 2) throughout the medical examination the veterinarians' attitude and handling techniques were always aimed at preventing stress and guaranteeing the best physical support possible. The videos were then evaluated for the number of fear and stress signs the subjects showed. The examined physiological variables were respiration (breath $/ \mathrm{min}$ ), heart rate (HR) and rectal temperature (RT). Physiological variables were analysed by t-Test for paired data while frequency of behavioural fear indicators by Wilcoxon signed-rank test. Rectal temperature was within range in both groups but significantly higher $(P<0.05)$ during LSH visit, while low head, lip licks and whale eye behaviours were significantly higher $(P<0.05)$ during TT visit. These results suggest that low stress handling decreases frequency of some fear-related behaviours and could improve the quality of human-dog interactions. Future research that aims to replicate and further investigate these results in a large canine population is required.

Pet Behaviour Science | 2017, Vol.4, 20 - 22

DOI: 10.21071/pbs.v0i4.10131

\begin{tabular}{|c|c|}
\hline $\begin{array}{l}\text { Bruno Scalia, Daniela Alberg } \\
\text { Panzera }\end{array}$ & ina and Michele \\
\hline Department of Veterinary Sciences, & Short Communication \\
\hline & \\
\hline & bru.scalia@hotmail.it \\
\hline Italy & Keywords: \\
\hline & dog; low-stress handling; \\
\hline & veterinary hospital; animal \\
\hline & welfare \\
\hline
\end{tabular}

This paper is based on a communication presented at '2017 Open Conference I Pet Behaviour Science' by the authors

Please, visit http://bit.ly/Scalia2017 to see the poster

\section{Introduction}

Low stress handling techniques or "Fear Free principles" in veterinary clinics are becoming an important research area aimed at improving small animal welfare (Yin, 2009; Overall, 2013; Lloyd 2017). If an animal experiences inadequate handling at the veterinary hospital, it is likely to become more fearful and difficult to handle during its next visits due to the poor association between the experience and the environment/personnel (classical conditioning) (Lloyd 2017). In a previous study $78.5 \%$ of dogs exhibited fear reactions during clinical visits, particularly on the examination table; those with only positive previous experiences were significantly less 'fearful' than those who had previously had a negative experience (Döring et al. 2009). Increased locomotor activity, panting, lip 
licking, tongue flicking, yawning, paw lifting, shaking, vocalization and lowered body posture have been identified as potential indicators of stress in dogs (Csoltova et al. 2017). Yin (2009) wrote a useful book to help veterinary staff take a low stress approach to companion animal care in the veterinary hospital. We hypothesized that physiological and behavioural stress indicators in dogs would increase more during traditional clinical examination than during "low stress handling" examination. Objective of this study was to compare a number of physiological and behavioural indicators using low stress handling techniques and traditional techniques.

\section{Methods}

Eight adult dogs (n.3 females and n. 5 males, aged from 3 to 10 years), used for clinical educational purposes at the Department of Animal Medicine and Surgery of the University of $X$, were enrolled in this study. Data were collected from March to May 2017. All dogs received routine healthcare and had been previously declared healthy by veterinarians. Moreover, they were unfamiliar with the examination room. To reduce the influence of individual variations, each dog was assigned to both the experimental and the control group (separated by a distance of $50 \pm 2$ days), thus all dogs in this study acted as their own controls. Group A $(\mathrm{N}=4)$ was assigned to the traditional technique (TT) at the first visit and to low stress handling (LSH) technique during the second visit, whereas group $\mathrm{B}$ $(\mathrm{N}=4)$ was assigned to LSH at the first visit and to TT during the second visit. The subjects were randomly allocated to either group. The testing procedures were always carried out by two veterinary medicine male students in the role of the veterinarian.

The study consisted of two clinical visits:

TT: The dog was kept on the examination table, under stationary conditions, using any required restraint. A standardized sequence of examination steps was performed: muzzle wearing, heart and lungs stethoscope examination, muzzle removal; eyes, ears and oral cavity examination; paws inspection; rectal temperature measurement; lateral recumbency positioning and abdominal area inspection; positioning the dog into a sit and simulated jugular venipuncture; sternal recumbency positioning and simulated cephalic vein catheter placement; simulated saphenous venipuncture. Throughout the examination, behaviours and heart rate (HR) of the dog were recorded.

LSH: Before the LSH clinical visit the dog was free to explore the environment for 5 minutes, while the researchers watched the dog's body language, avoided direct eye contact and greeted it correctly; some treats were tossed on the floor. When no signs of fear were detected, the researcher approached the dog in order to begin the low stress handling visit. A soft towel had been laid on the table and tasty treats were available. The sequence of the examination was the same as for the TT visit, except that the veterinarians' attitude and the handling techniques were aimed at preventing fear and stress and guaranteeing the best physical support possible. After the visit the dog was free to further investigate the environment, received some other treats and was invited to playing activities for approximately 5 minutes.

The examined physiological variables were respiration (breath/min), heart rate (HR) and rectal temperature (RT). Rectal measurements were taken with a digital thermometer. The thermometer was gently inserted into the rectum for a length of about $2 \mathrm{~cm}$. The thermometer emitted an acoustic signal when the attained temperature remained stable, after approximately 1 minute. The thermometer was disinfected after each sampling. Seven behaviours ("low head position", "panting", "lip licks", "yawns", "whale eye", "hypervigilance", "urination and defecation") were logged as the number of events per $15 \mathrm{~min}$. All behavioural data was collected by video recording. Physiological variables were analysed by $\mathrm{t}$ Test for paired data while frequency of behavioural fear indicators by Wilcoxon signed-rank test using Statistica 8 software (Statsoft Inc., Tulsa, OK, USA). The significance level was set at $\mathrm{P}<0.05$.

\section{Results}

A significant difference was found between groups on rectal temperature $(\mathrm{t}=2.942 \mathrm{df}=7, P<0.05)$, low head $(\mathrm{W}=21, P<0.05)$, lip licks $(\mathrm{W}=21, P<0.05)$ and whale eye behaviours $(\mathrm{W}=36, P<0.05)$. Rectal temperature was within range in both groups but significantly higher 
during LSH visit than during TT visit $\left(38.78 \pm 0.33^{\circ} \mathrm{C}\right.$ and $38.45 \pm 0.37^{\circ} \mathrm{C}$ respectively), while low head, lip licks and whale eye behaviours were significantly higher during TT visit.

\section{Conclusions}

The results of this preliminary study indicate that "low stress handling" significantly affected rectal temperature and low head posture, lip licks and whale eye behaviours. The influence on rectal temperature could be related to a different temperature perception through the examination table (lower table temperature during TT than during LSH visits) but many conditions, including digestion, peristaltic movements, fecal masses and physical activity may affect rectal temperature (Rexroat et al. 1999; Rizzo et al. 2017). Lip licking has been previously related to salivary cortisol concentrations in hospitalized dogs (Hekman et al. 2012) and could be useful for the evaluation of acute stress levels in a social context (Beerda et al. 1998). Limitations to the findings of this study are the small number of subjects, the adult age of enrolled dogs and the absence of interaction with an owner. Future research that aims to replicate and further investigate these results in a large canine population is required. In conclusion, our results suggest that "low stress handling" decreases frequency of behaviours related to fear and could improve welfare related to human interactions in adult dogs.

\section{References}

Beerda, B., Schilderm M.B.H., van Hooff, J.A.R.A.M., de Vries, H.W., Mol, J.A. 1998. Behavioural, saliva cortisol and heart rate responses to different types of stimuli in dogs. Applied Animal Behavior Science 58: 365-381.

Csoltova, E., Martineau, M., Boissy, A., Gilbert, C. 2017. Behavioral and physiological reactions in dogs to a veterinary examination: Owner-dog interactions improve canine well being. Physiology and Behavior 177: 270-281.
Döring, D., Roscher, A., Scheipl, F., Küchenhoff, H., Erhard, M.H. 2009. Fear-related behaviour of dogs in veterinary practice. Veterinary Journal 182: 38-43.

Hekman, J.P., Zaras, A.Z., Dreschel, N.A. 2012. Salivary cortisol concentrations and behavior in a population of healthy dogs hospitalized for elective procedures. Applied Animal Behavior Science 141: 149-157.

Lloyd, J.K.F. 2017. Minimising Stress for Patients in the Veterinary Hospital: Why It Is Important and What Can Be Done about It. Veterinary Sciences 4(2) doi:10.3390/vetsci4020022.

Overall, K.L. 2013. Fear Factor: Is Routine Veterinary Care Contributing to Lifelong Patient Anxiety? MAGAZINE September 1.

Rexroat, J., Benish, K., Fraden, J. 1999. Clinical accuracy of Vet-TempTM instant ear thermometer: Comparative study with dogs and cats. Advances Monitor Corporation1-4.

Rizzo, M., Arfuso, F., Alberghina, D., Giudice, E., Gianesella, M., Piccione, G. 2017. Monitoring changes in body surface temperature associated with treadmill exercise in dogs by use of infrared methodology. Journal of Termal Biology 69:64-68.

Yin, S. Low Stress Handling, Restraint and Behavior Modification of Dogs and Cats: Techniques for Patients Who Love Their Visits; CattleDog Publishing: Davis, CA, USA, 2009.

\section{Ccreative}

This paper has been published by Pet Behaviour Science under a Creative Commons license 4.0 Non-comercial - Share Alike - Attribution

As an open access journal, it is free of charges for both authors and readers

www.petbehaviourscience.org 